\title{
Facilitative effects of stimulus familiarization on paired associates verbal learning'
}

\author{
HILDEGARD CORBET AND MARILYN E. MARSHALL \\ CARLETON UNIVERSITY, OTTAWA, CANADA
}

With girls as Ss, a paced verbal paired associate task was reliably facilitated after relevant stimulus familiarization. Discrimination familiarization resulted in significantly better performance than observation familiarization. Neither relevancy nor kind of familiarization affected the performance of boys.

When Ss are familiarized with verbal items which appear subsequently as response members in a paired associate (PA) task, performance on the latter task has been shown to be facilitated (e.g., Underwood \& Schulz, 1960). Under similar conditions, however, verbal stimulus familiarization has not yielded reliable proactive facilitation (e.g., Goss \& Nodine, 1965) unless stimulus items are pronounced during both familiarization and test tasks (Gannon \& Noble, 1961), or unless proaction is defined by the comparison of familiarization groups with a no-familiarization control (Orlowsky \& Walsh, 1967).

If familiarization with stimuli prior to a PA task is conceived as serving $\mathrm{S}$ primarily in allowing him to predifferentiate test task items, then the possible facilitative effects of such familiarization should be expected to emerge only if criterion items are initially difficult to discriminate. In the present study, an attempt was made to set optimal conditions for the emergence of the stimulus familiarization effect by employing stimuli of low meaningfulness and high auditory and formal similarity, and by constructing stimulus items such that Ss would be forced to scan all or most of the stimulus in order to isolate efficient functional cues.

It was argued that for familiarization to be effective, the attention of Ss during the familiarization task should be directed to the kind of stimulus encoding which is deemed most necessary to the mastery of the test task. To evaluate the latter argument, a familiarization procedure which demanded the discrimination of test stimuli from other verbal items was compared with familiarization involving mere observation of the test stimuli.

Under the conditions just described, facilitative stimulus familiarization effects have been demonstrated using college students as Ss (Corbet, 1966). The present experiment was designed to verify the earlier research using children as Ss.

Method

Subjects were 60 male and 60 female sixth-grade children. At the beginning of the experiment, all Ss were given five warm-up trials of PA practice with a list of three closely associated noun pairs. Subjects in observation (O) and discrimination (D) groups then expe- rienced each stimulus in 12 double exposures. During the first half of each double exposure the stimulus appeared alone on a memory drum for 2 sec; during the second half of the double exposure, $O$ groups visually experienced each stimulus singly on a card, whereas D groups had to select the just-observed item from four similar stimuli on a card. All Ss were instructed to circle the critical item during the second 2-sec interval. Relevant familiarization (RF) groups were familiarized with stimuli which also occurred later on the test task (ZUK, SOQ, ZOQ, ZOG); irrelevant familiarization (IF) groups were familiarized with stimuli different from those of the test task (VEB, VED, WYB, VYB), while an additional control (C) received no familiarization prior to the test task.

The test task proceeded by the paced PA anticipation method. Subjects vocally anticipated responses (numerals 3-6) but, as during familiarization, stimuli were not pronounced. Twenty PA trials were given.

\section{Results}

The effects of relevance of familiarization (relevant vs irrelevant), kind of familiarization (observation vs discrimination), IQ ("low" or "high," i.e., 89-117 vs 118-172), and sex were evaluated statistically in a $2^{4}$ factorial design. The factorial analysis applied to correct test task responses yielded significant interactions between sex and relevancy $(F=5.63, d f=1 / 80$, $p<.05)$, sex and IQ $(F=10.75$, df $=1 / 80, p<.01)$, and between IQ and kind of familiarization $(F=6.68$, $\mathrm{df}=$ $1 / 80, p<.05)$. Separate factorial analyses were subsequently conducted on the data for boys and girls.

For boys, the only significant finding was the better performance of the high as compared with the low IQ. group $(F=9.91$, $d f=1 / 40, p<.01)$. As the interest of this experiment lay in the possible facilitative effects of relevant stimulus familiarization, the data of the boys received no further attention.

In the data of the girls, however, RF treatments $(\bar{X}=51.5)$ led to more correct responses on the test task than did IF $(\overline{\mathrm{X}}=44.5)$ or $\mathrm{C}(\overline{\mathrm{X}}=40.2)$ treatments. Additionally, D groups $(\bar{X}=51.2)$ performed better on the subsequent paired associate task than did $O(\bar{X}=44.8)$ or $\mathrm{C}$ groups. A factorial analysis applied to these data revealed significant main effects for both relevancy $(\mathrm{F}=8.36, \mathrm{df}=1 / 40, \mathrm{p}<.01)$ and kind $(\mathrm{F}=7.02, \mathrm{df}=1 / 40$, $p<.025)$ of familiarization. The main effect of $I G$ was not significant, nor did any interaction prove statistically reliable.

When the performance of the combined RF groups was compared with that of the $C$ group by means of a $t$ test, the difference was statistically significant 
$(t=6.3, d f=34, p<.01)$, while the superiority of combined IF groups to group $C$ did not prove statistically reliable. Discussion

Whether or not the significant difference between the performance of male and female children is attributable to an interaction between $\mathrm{E}$ sex and $\mathrm{S}$ sex, or to different learning behavior in boys and girls of the same age will have to be investigated in further studies. A similar, though less marked sex difference was observed with college students as Ss (Corbet, 1966), and it is suggested that the evaluation of sex differences be regarded as essential in experiments of this nature.

The most provocative finding of the present study is the statistically reliable effect of RF for girls, and it is suggested that this usually elusive phenomenon emerged in the present experiment by virtue of: (a) the critically difficult-to-discriminate stimuli employed; (b) the already consolidated and available responses of the test task; (c) the control of task-general variables through a prior warm-up task; and (d) the separate analysis by sex.

The significant overall superiority of relevant over irrelevant familiarization procedures must be attributed to specific stimulus attributes, and this fact suggests that under the special conditions prescribed by the present design, verbal PA learning may be conceived as a three-stage process-a process involving response consolidation and associative hookup, as postulated and demonstrated by Underwood \& Schulz (1960), and an additional stimulus differentiation phase which other authors (e.g., Battig, Williams, \& Williams, 1962) have suggested previously. There is a substantial literature which shows the reliable superiority of relevant stimulus pre-training when the stimuli of a PA criterion task are nonverbal in nature (e.g., Cantor, 1955; Cantor, 1965; Marshall, 1965), and a continuity between nonverbal and verbal stimulus situations is here suggested as a reasonable and potentially fruitful hypothesis. The relative importance of each of the stages in PA learning with stimuli of whatever nature should be found to be determined by the stimulus, response, and associative attributes peculiar to the materials and task employed.

The superior facilitative effects of discrimination over observation procedures are reflected in both $\mathrm{RF}$ and IF groups. This superiority cannot, therefore, be attributed to specific stimulus sources, for Ss in IF groups had not seen the test task stimuli prior to the onset of the test task. Although irrelevant and test task stimuli differed in unique respects, however, they did share certain common stimulus class charac- teristics, and could thus be considered comparable in the sense of demanding of Ss the same kinds of differentiation strategies. Thus, the difference in performance between $D$ and $O$ groups most likely arises from the differential demands placed upon Ss during $\mathrm{D}$ and $\mathrm{O}$ familiarization and, as suggested by Orlowsky \& Walsh (1967), from the relation between the demands of familiarization and test tasks. While Ss in O groups saw verbal stimuli of the test task class as often as did the Ss in D groups, and while Ss in the O groups may have used their own-and probably varied-strategies to deal with the verbal material during familiarization, they were not forced, as were Ss in D groups, to learn and to practice the strategies by which verbal units of the test task class subsequently could more easily be discriminated.

In conclusion, the data of the present experiment suggest that facilitation through relevant stimulus familiarization may arise from two measurable sources. The first source resides in the identity of specific stimulus characteristics from training to test task, and the second in the similarity of demands forced upon the Ss for strategy formation during training and test tasks.

\section{References}

BATTIG, W. F., WILliaMS, J. M., \& WILliaMS, J. G. Transfer from verbal-discrimination to paired-associate learning. J. exp. Psychol., 1962, 63, 258-268.

CANTOR, G. N. Effects of three types of pretraining on discrimination learning in preschool children. J. exp. Psychol., 1955, 49, 339-42.

CANTOR, JOAN $\mathrm{H}$. Transfer of stimulus pretraining in motor pairedassociate and discrimination learning tasks. In L. P. Lipsitt and C. C. Spiker (Eds.) Advances in child development and behavior. New York: Academic Press, 1956, Vol. 2, pp. 19-58.

CORBET, HILDEGARD. Stimulus familiarization prior to paced verbal paired-associates learning. Unpublished M. A. thesis, Carleton University, 1966.

CORBET, HILDEGARD \& MARSHALL, MARILYN E. Facilitation of a verbal paired-associates tests after stimulus familiarization. Abstract in Canad. Psychol., 1967, 8a, 159.

GANNON, D. R., \& NOBLE, C. E. Familiarization (n) as a stimulus factor in PA verbal learning. $J$. exp. Psychol, 1961, 62, 1423.

GOSS, A. E., \& NODINE, C. F. Paired-associates learning. New York: Academic Press, 1965.

MARSHALL, MARILYN E. Note on motor performance following four amounts of relevant verbal pretraining. Percept. Mot. Skills, 1965, 20, 628.

ORLOWSKY, W. J., \& WALSH, J. F. The effect of stimulus familiarization procedure on paired-associate verbal learning. Psychon. Sci. $1967,8,435-436$.

UNDERWOOD, J. B., \& SCHULZ, R. W. Meaningfulness and verbal learning. New York: Lippincott, 1960.

\section{Note}

1. A paper based on the results of this study was read at a meeting of the Canadian Psychological Association. (See Corbet \& Marshall, 1967). We are grateful to $\mathrm{Dr}$. T. J. Ryan for suggestions and criticism. 